Paper presented at NN97, Sixth International Conference on Nucleus-Nucleus Collisions, Gatlinburg, Tennessee, USA, June 2-6 1997. To be published in Nucl. Phys. A.

\title{
Manifestation of halo size in scattering and reactions
}

\author{
J. A. Tostevin*a, R. C. Johnson and J. S. Al-Khalili \\ a Department of Physics, University of Surrey, Guildford, Surrey, GU2 5XH, U.K.
}

Total reaction cross section and elastic scattering angular distribution calculations are discussed for composite (halo) nuclei with well developed core and valence particle structures. We review the basis of few-body calculations of reaction cross sections and use a simple binary cluster model to clarify the inadequacy of optical limit Glauber calculations for determining the sizes of correlated nuclear systems. An outstanding discrepancy in the case of the ${ }^{6} \mathrm{Li}$ and ${ }^{6} \mathrm{He}$ systems is discussed. We show that under certain conditions complementary information on halo sizes could be obtained from precision elastic scattering measurements at lower energies.

\section{INTRODUCTION}

The matter radii of the least bound nucleon(s) of halo nuclei are accurate indicators of underlying ground state structures. Evaluating theories for determining these halo sizes is therefore of considerable importance. The widths of measured momentum distributions following the breakup of halo nuclei also offer a clear qualitative signature of the spatial distribution of the halo particles. However, the complexity of the reaction mechanism, including the role of final state interactions, has meant that momentum widths have yet to produce reliable quantitative information on halo sizes. Progress in this area is a significant theoretical challenge.

Most published tabulations of halo nucleus sizes have been deduced by comparing high energy interaction cross section measurements [1,2] with model calculations which use the optical limit (OL) of Glauber theory $[3,4]$. In this theoretical limit the structure of the composite projectile enters only through its single particle density, appearing to offer a direct and rather model independent measure of this distribution. However, the OL approximation is appropriate only to the extent that the projectile nucleons can be considered to move independently and are assumed uncorrelated except for the fact that they share a common volume of space [5].

The most dramatic feature of halo nuclei is precisely their loosely bound few-body character, the strong spatial localisation of the core nucleons, and the delocalisation of the halo particles. Recent analyses [6-8] have shown that an explicit treatment of this correlated few-body nature is important in calculations of reaction cross sections. These lead to smaller cross sections than obtained from the OL theory with significant implications for the deduced rms radius of the nucleus and the halo. It follows that estimates of the

*The financial support of the United Kingdom Engineering and Physical Sciences Research Council (EPSRC) in the form of Grant No. GR/J95867 is gratefully acknowledged 
matter radii of halo nuclei, even from high energy data, are intrinsically model dependent and require the use of realistic theoretical wavefunctions for the few-body structures $[6,7]$.

Here a simple binary cluster model is introduced and used to clarify both this physical picture and the sensitivity of calculated cross sections to the clustering of nucleons. The model allows us to construct composite nuclei of quite different structures but with a common single particle density. It follows that all such structures would predict the same cross section in the OL theory. We show that when the projectile structure is included explicitly, these different configurations lead to quite different reaction cross sections, which depend on the underlying binary decomposition, and the degree of nucleon clusterisation.

Such total cross section analyses at very high energies are increasingly giving way to exclusive and differential cross section measurements; many at energies of between 30 and $100 \mathrm{MeV} /$ nucleon. At these lower energies the reaction mechanisms are more complex and an explicit treatment of breakup and inelastic degrees of freedom is then essential. Nevertheless even simple considerations, such as folding model ideas, lead us to expect that composite nucleus-target elastic scattering observables should manifest some signs of the size of the projectile. The importance of coupling to the breakup continuum for the extended and loosely bound halo systems means however that folding model ideas are an inappropriate starting point. What then is the nature of this sensitivity for halo nuclei?

We show that under certain conditions the elastic scattering of a halo nucleus from a stable target can give simple direct evidence for the structure of the halo, even in the presence of strong coupling between the halo ground state and the low energy breakup continuum [9]. This coupling to elastic breakup channels is in fact essential to the analysis, which, in elastic scattering, will be useful when the ratio of the projectile's halo mass to its core mass is small.

Underlying all of the theoretical ideas presented is the use of the adiabatic approximation for composite systems. In the case of the reaction cross section analysis the additional eikonal (Glauber) approximation of straight line trajectories is made.

\section{GLAUBER THEORY CALCULATIONS FOR COMPOSITE NUCLEI}

For composite nuclei all Glauber theoretical approaches start from the approximation that the internal motions of the particles within the projectile and target are assumed slow compared to the relative motion of the centres of mass of the projectile and target; and so can be treated adiabatically [3]. Additional approximations are then made. In halo nucleus induced reactions, where the projectile's internal degrees of freedom are of particular interest it is usual to treat the projectile structure explicitly and the mass $A_{t}$ target as presenting a scattering centre to each projectile constituent. The position coordinates of these constituents is assumed frozen during the transit of the projectile past the target.

At the energies $(\approx 800 \mathrm{MeV} / \mathrm{A})$ of interaction cross section measurements the adiabatic approximation is certainly reliable. For an $n$-body composite projectile, with ground state $\left|\Phi_{0}^{(n)}\right\rangle$, the projectile-target elastic $S$-matrix is

$S_{p}^{(n)}(b)=\left\langle\Phi_{0}^{(n)}\left|S_{1}\left(b_{1}\right) S_{2}\left(b_{2}\right) \ldots S_{n}\left(b_{n}\right)\right| \Phi_{0}^{(n)}\right\rangle$.

Each $S_{j}\left(b_{j}\right)$ is the eikonal approximation to the elastic $S$-matrix for projectile constituent $j$ 
scattering independently from the target [3] and is a function of its own impact parameter $b_{j} . S_{p}^{(n)}$, the projectile ground state matrix element of a many-body operator, is expected to be sensitive to properties of the ground state beyond its single particle density.

\subsection{Optical limit approximation}

The OL approximation to $S_{p}$ neglects entirely any correlations between constituents in the projectile or target. The calculation of $S_{p}$, and the reaction cross section, then involves only the projectile and target ground state densities $\rho_{p}$ and $\rho_{t}$. Explicitly,

$S_{p}^{O L}(b)=\exp \left[i \mathcal{O}^{p t}(b)\right]$,

where $\mathcal{O}^{p t}(b)$ is the overlap of the two densities with a nucleon-nucleon (NN) formfactor integrated along the assumed straight line path of the projectile's c.m. at impact parameter $b$. Expressing the projectile-target separation in cylindrical coordinates $\boldsymbol{R}=\left(\boldsymbol{b}, R_{3}\right)$, with the $z \equiv 3$ axis chosen along the incident beam direction, then

$\mathcal{O}^{p t}(b)=\int_{-\infty}^{\infty} d R_{3} \int d \boldsymbol{r}_{1} \int d \boldsymbol{r}_{2} \rho_{p}\left(r_{1}\right) \rho_{t}\left(r_{2}\right) \hat{f}_{N N}\left(\left|\boldsymbol{R}+\boldsymbol{r}_{1}-\boldsymbol{r}_{2}\right|\right)$

For an absorptive zero-range NN amplitude and a $T=0$ target, then

$\hat{f}_{N N}(\boldsymbol{r})=\left(i \bar{\sigma}_{N N} / 2\right) \delta(\boldsymbol{r})$

where $\bar{\sigma}_{N N}$ is the average of the free $\mathrm{nn}$ and $\mathrm{np}$ total cross sections at the energy of interest.

\subsection{Few-body description}

The approximation of independent particle motion underlying the OL approach is inconsistent with the loosely bound few-body (FB) character of halo nuclei. Such systems require an explicit treatment of this correlated FB nature [6,7]. In the FB Glauber picture, with its adiabatic basis, the scattering must be calculated for each configuration of the constituents and only then should the resulting scattering amplitudes be averaged over all contributing configurations. That is, Eq. (1) should be evaluated explicitly for the few-body system. Since the constituent core- and valence particle-target two-body systems are themselves well localised, these binary subsystems can be treated using the OL theory. For an assumed two-body composite projectile, with a valence and core cluster with relative motion wavefunction $\Phi_{0}^{(2)}$, then $[6,7]$

$S_{p}^{F B}(b)=\left\langle\Phi_{0}^{(2)}\left|S_{c}^{O L}\left(b_{c}\right) S_{v}^{O L}\left(b_{v}\right)\right| \Phi_{0}^{(2)}\right\rangle$

Evaluation of both the FB $\left(S_{p}^{F B}\right)$ and OL $\left(S_{p}^{O L}\right) S$-matrices, when using the most realistic theoretical inputs, becomes a reasonably involved numerical task at this point. The following therefore presents a simple model description, involving Gaussian densities, which allows the consequences of the FB formulation to be exposed more clearly. In this analysis the zero range approximation to $\hat{f}_{N N}$ is quite adequate to clarify the essential differences calculated in the FB and OL schemes. 


\subsection{Simple binary cluster structure model}

We consider a composite projectile of mass $A_{p}$ as consisting of valence and core clusters, of masses $A_{v}$ and $A_{c}$, bound in a state of relative motion, Figure 1. To be definite we assume $A_{c} \geq A_{v}$. For simplicity the internal densities of the clusters are described by single Gaussian functions with ranges $\alpha_{c}$ and $\alpha_{v}$,

$\rho_{c}(r)=A_{c} g^{(3)}\left(\alpha_{c}, r\right), \quad \rho_{v}(r)=A_{v} g^{(3)}\left(\alpha_{v}, r\right)$,

where $g^{(3)}$ is the normalised 3-dimensional Gaussian function

$g^{(3)}(\gamma, r)=(\sqrt{\pi} \gamma)^{-3} \exp \left(-r^{2} / \gamma^{2}\right), \quad \int d \boldsymbol{r} g^{(3)}(\gamma, r)=1 \quad, \quad\left\langle r^{2}\right\rangle=3 \gamma^{2} / 2$

If we also assume that the relative motion wavefunction $\Phi_{0}^{(2)}(\boldsymbol{r})$ of the two clusters is a $0 s$ oscillator state of range parameter $\alpha$ then of course

$\left|\Phi_{0}^{(2)}(\boldsymbol{r})\right|^{2}=g^{(3)}(\alpha, r)$

with mean squared cluster separation of $\left\langle r^{2}\right\rangle=3 \alpha^{2} / 2$.

While simple this model is very flexible. Moreover it allows us to construct explicitly the single-particle density of the composite, needed for OL calculations. Upon convoluting the intrinsic cluster densities with their c.m. motions about the c.m. of the projectile, the composite projectile density is

$\rho_{p}(r)=A_{c} g^{(3)}\left(\hat{\alpha}_{c}, r\right)+A_{v} g^{(3)}\left(\hat{\alpha}_{v}, r\right)$

with range parameters

$$
\begin{aligned}
& \hat{\alpha}_{v}^{2}=\alpha_{v}^{2}+\left(\frac{A_{c} \alpha}{A_{v}+A_{c}}\right)^{2}, \\
& \hat{\alpha}_{c}^{2}=\alpha_{c}^{2}+\left(\frac{A_{v} \alpha}{A_{v}+A_{c}}\right)^{2} .
\end{aligned}
$$

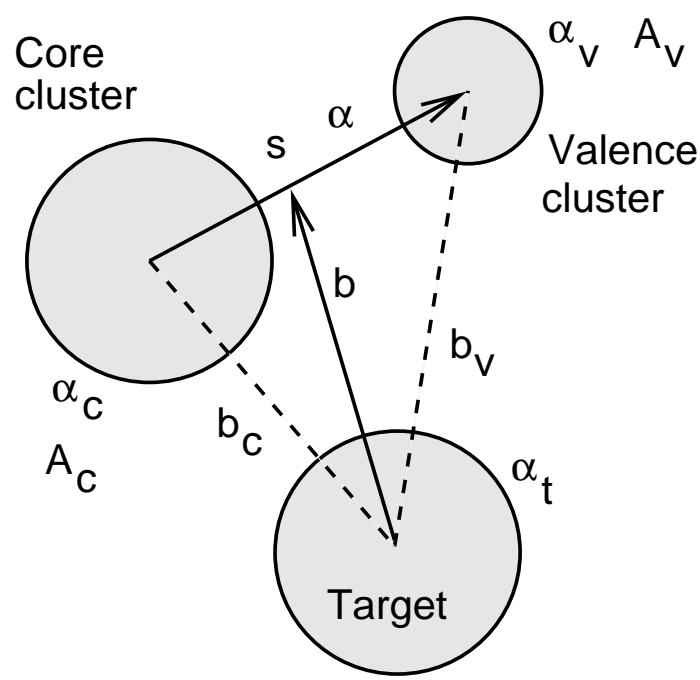

Figure 1. The two cluster projectile and target coordinates

The mean squared radius of the composite $\left\langle r^{2}\right\rangle_{p}$ satisfies

$A_{p}\left\langle r^{2}\right\rangle_{p}=A_{c}\left\langle r^{2}\right\rangle_{c}+A_{v}\left\langle r^{2}\right\rangle_{v}+\left(A_{v} A_{c} / A_{p}\right)\left\langle r^{2}\right\rangle=(3 / 2)\left(A_{v} \hat{\alpha}_{v}^{2}+A_{c} \hat{\alpha}_{c}^{2}\right)$.

The model thus derives a projectile density with distinct components due to the valence and core clusters, containing different numbers of nucleons, and with different spatial extensions. Such simple two component forms have been used widely to model density distributions of light exotic nuclei and also used as input to OL calculations of reaction cross sections to deduce matter radii and valence particle properties.

We note however that a particular projectile single particle density, specified by a given $\left(A_{c}, A_{v}\right)$ mass split and choice of the two component ranges $\left(\hat{\alpha}_{c}, \hat{\alpha}_{v}\right)$, does not define the underlying structure of the projectile. Eq. (10) shows that any chosen $\hat{\alpha}_{v}$ and $\hat{\alpha}_{c}$ are 
consistent with an infinite number of two cluster structures with different cluster rms sizes and separations. Specifically, for a fixed projectile density, $\alpha$ can take on all values from zero to an upper limit $\alpha_{\max }$, where

$\alpha_{\max }=\min \left[\left(A_{p} / A_{c}\right) \hat{\alpha}_{v},\left(A_{p} / A_{v}\right) \hat{\alpha}_{c}\right]$,

at which limit one or other of the internal cluster densities is pointlike. Similarly, if one of the original clusters is pointlike, e.g. $\alpha_{v}=0$, then fixing $\hat{\alpha}_{v}$ and $\hat{\alpha}_{c}$ uniquely determines $\alpha$ and hence $\alpha_{c}$. We discuss these two cases in the following.

\subsection{Reaction cross section calculations}

Assuming the target nucleus is also described by a Gaussian density of range $\alpha_{t}$, then the OL and FB expressions for $S_{p}$ take particularly simple forms. Eqs. (2) and (3) give

$S_{p}^{O L}(b)=\exp \left[-\frac{\bar{\sigma}_{N N} A_{t}}{2}\left(A_{c} g^{(2)}\left(\hat{\alpha}_{c t}, b\right)+A_{v} g^{(2)}\left(\hat{\alpha}_{v t}, b\right)\right)\right]$

where $g^{(2)}(\gamma, b)=(\sqrt{\pi} \gamma)^{-2} \exp \left(-b^{2} / \gamma^{2}\right)$ is a normalised two-dimensional Gaussian, $\hat{\alpha}_{v t}^{2}=$ $\hat{\alpha}_{v}^{2}+\alpha_{t}^{2}$, and similarly for $\hat{\alpha}_{c t}^{2}$. The FB $S$-matrix, Eq. (5), is

$S_{p}^{F B}(b)=\int d^{2} \boldsymbol{s} g^{(2)}(\alpha, s) \exp \left[-\frac{\bar{\sigma}_{N N} A_{t}}{2}\left(A_{c} g^{(2)}\left(\alpha_{c t}, b_{c}\right)+A_{v} g^{(2)}\left(\alpha_{v t}, b_{v}\right)\right)\right]$

with $\boldsymbol{s}$ the projection of $\boldsymbol{r}$ in the impact parameter plane and $b_{c}=\left|\boldsymbol{b}-A_{v} \boldsymbol{s} / A_{p}\right|$ and $b_{v}=\left|\boldsymbol{b}+A_{c} \boldsymbol{s} / A_{p}\right|$ are the impact parameters of the core and valence clusters, Figure 1 . In this case $\alpha_{v t}^{2}=\alpha_{v}^{2}+\alpha_{t}^{2}$, etc., arising from the convolutions of the free cluster and target densities. We note that $S_{p}^{O L}=S_{p}^{F B}$ only in the limit that $\alpha \rightarrow 0$.

We calculate the reaction cross sections using

$\sigma_{R}=2 \pi \int_{0}^{\infty} d b b\left[1-\left|S_{p}(b)\right|^{2}\right]$.

The value $\bar{\sigma}_{N N}=4.11 \mathrm{fm}^{2}$, appropriate to $800 \mathrm{MeV} / \mathrm{A}$ incident energy [10], is assumed. The results in a representative case of a mass $A_{p}=10$ projectile $\left(\left\langle r^{2}\right\rangle_{p}^{1 / 2}=3.1 \mathrm{fm}\right)$ with $\left(A_{c}, A_{v}\right)=(8,2)$ and a mass $A_{t}=12$ target $\left(\left\langle r^{2}\right\rangle_{t}^{1 / 2}=2.32 \mathrm{fm}\right)$ are presented in Figure 2 . We have fixed the range parameters $\hat{\alpha}_{v}$ and $\hat{\alpha}_{c}$ such that $A_{v} \hat{\alpha}_{v}^{2}=A_{c} \hat{\alpha}_{c}^{2}$, see Eq. (11), so that the core and valence particles make equal contributions to the projectile rms matter radius. This ensures due emphasis is given to the valence particles, but still underestimates the valence particle contributions compared to realistic halo structures.

The parameters above fix uniquely the projectile single particle density, the $S_{p}^{O L}$, and hence the reaction cross section calculated in the OL theory. This is shown by the dashed line in Figure 2, and is independent of the details of the underlying cluster sizes and separations. The actual two component density is shown as an inset to the figure. This two component density is consistent with a range of structures as was expressed by Eqs. (10) and (12). The cross sections calculated in the FB approach, from $S_{p}^{F B}$, are shown by the solid symbols and solid line in the figure as a function of the assumed rms separation of the centres of mass of the two clusters. As the graphics indicate, the valence and core cluster internal densities have to become increasingly localised at large rms separations to maintain the fixed projectile density. The limiting situation, where the valence cluster 
has become pointlike, is reminiscent of early point dineutron models of two neutron halo systems. In this limit the cross sections calculated using the FB and OL theories are very significantly different.

These calculations show clearly that, for composite systems, there is no model independent relationship between the calculated (or measured) reaction cross section and the projectile single particle density; since all calculated cross sections in the figure are consistent with the same density. The structures differ in the way the nucleons are correlated within the projectile and which are treated explicitly within the few-body framework. Structure or density information extracted from reaction cross section data therefore relies heavily upon an assumed theoretical cluster structure and associated few-body wavefunction.

As was already noted, if one of the clusters is intrinsically pointlike, such as a single nucleon then, in our simple model, a given projectile density does indeed correspond to a unique rms separation for the valence and core clusters. This does not imply however that the FB and OL theories agree in this single nucleon halo case. Figure 3 shows calculations of the OL (dashed curve) and FB (solid curve) cross sections for $\left(A_{c}, A_{v}\right)=(9,1)$ with $\left\langle r^{2}\right\rangle_{c}^{1 / 2}=2.30 \mathrm{fm}$, as a function of the rms radius of the projectile; for the same $A_{t}=12$ target as above. Again the OL overpredicts the cross section when compared with the FB theory and, in comparisons with any measured cross section datum, the OL will underestimate the extension of the composite system.

\subsection{Status of $A_{p}=6$ calculations}

The model calculations above reveal the sensitivity of reaction cross sections to the degree of nucleon clustering within the composite. This may be significant in the context of cross section calculations for ${ }^{6} \mathrm{He}$ and ${ }^{6} \mathrm{Li}$ on ${ }^{12} \mathrm{C}$ at $800 \mathrm{MeV} / \mathrm{A}$. For these three-body 
systems then

$S_{p}^{F B}(b)=\left\langle\Phi_{0}^{(3)}\left|S_{\alpha}^{O L}\left(b_{\alpha}\right) S_{N}^{O L}\left(b_{1}\right) S_{N}^{O L}\left(b_{2}\right)\right| \Phi_{0}^{(3)}\right\rangle$

where the $\Phi_{0}^{(3)}$ are three-body $\alpha+\mathrm{N}+\mathrm{N}$ wavefunctions. In these quantitative studies we use the finite range NN formfactors of Ray [11] in Eq. (3). We take $\left\langle r^{2}\right\rangle_{\alpha}^{1 / 2}=1.50 \mathrm{fm}$. The results are collected in Figure 4. The horizontal lines show the measured interaction cross sections (solid), and associated error bands (dashed), for ${ }^{6} \mathrm{He}$ (722 $\pm 5 \mathrm{mb}$ [1], upper) and ${ }^{6} \mathrm{Li}(688 \pm 10 \mathrm{mb}[12]$, lower). These differ by $34 \mathrm{mb}$ but with a significant error.

Calculations for different three-body wavefunctions are shown by the solid $\left({ }^{6} \mathrm{He}\right)$ and open $\left({ }^{6} \mathrm{Li}\right)$ symbols. The ${ }^{6} \mathrm{He}$ calculations are those reported in [8]. From left to right the ${ }^{6} \mathrm{Li}$ calculations use the OE1D, OE3B, I4 and H3 model wavefunctions of Thompson [13]. For ${ }^{6} \mathrm{He}$ the wavefunction (FC6) consistent with the measured cross section has the correct two neutron separation energy and predicts a ${ }^{6} \mathrm{He}$ rms radius of approximately $2.54 \mathrm{fm}$. For ${ }^{6} \mathrm{Li}$ however the two theoretical wavefunctions with the correct binding energy (OE3B and I4) cluster in the region of the matter radius $2.44 \mathrm{fm}$, consistent with that from the ${ }^{6} \mathrm{Li}$ charge radius [14], but have calculated cross sections close to that of ${ }^{6} \mathrm{He}$.

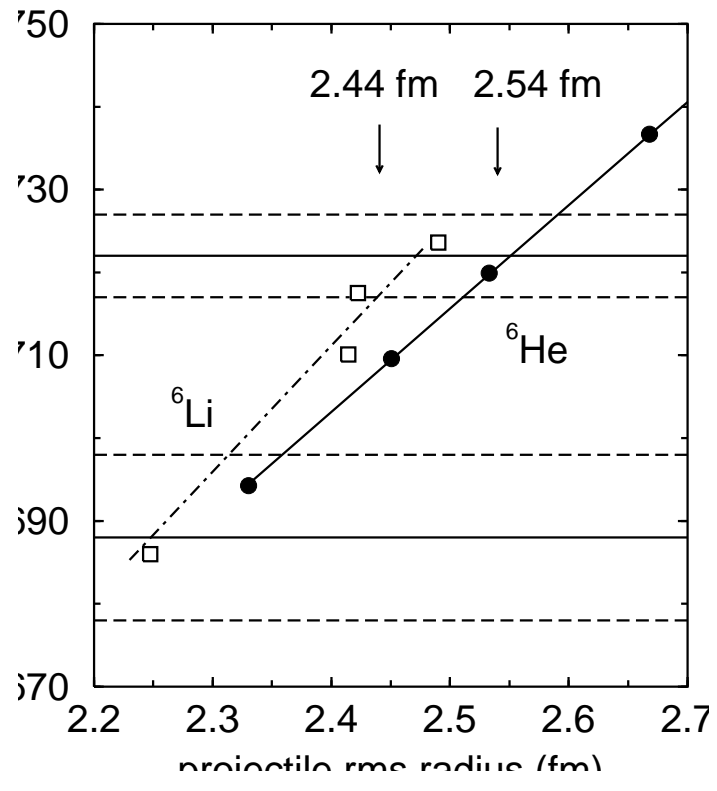

Figure 4. Reaction cross sections for ${ }^{6} \mathrm{He}$ and ${ }^{6} \mathrm{Li}$ as a function projectile rms radius.

The ${ }^{6} \mathrm{Li}$ calculation with the largest cross section (H3) is underbound and that with the smaller cross section (OE1D), which overlaps the experimental value, is achieved only by overbinding the system. It appears therefore that theoretical ${ }^{6} \mathrm{Li}$ and ${ }^{6} \mathrm{He}$ wavefunctions with the correct separation energies are much more similar than the measured interaction cross sections would suggest. This is disturbing since the stable ${ }^{6} \mathrm{Li}$ system is an important test case for both structure and reaction calculations. A possible explanation, given the model calculations above, is that the expected np clustering in ${ }^{6} \mathrm{Li}$ is not adequately represented, or fully converged, within the three-body wavefunctions used. Further theoretical work is needed here. A more accurate experimental cross section measurement in the case of ${ }^{6} \mathrm{Li}$ would also be extremely useful.

\section{ELASTIC SCATTERING AND BREAKUP OF HALO NUCLEI}

We now turn our attention to calculations of the elastic scattering of an assumed twobody projectile with core mass $A_{c}$ and valence mass $A_{v}$ by a target nucleus of mass $A_{t}$. We will assume: $(i)$ that the core-target interaction $V_{c}$ is effectively much stronger than the valence-target interaction $V_{v}$, and $(i i)$ that the relative motion of the core and valence particles can be treated adiabatically. For such a three-body system full quantum 
mechanical calculations, which use the adiabatic assumption but not the the additional assumption of the dominance of $V_{c}$, can be performed [15] to assess this approximation.

Our condition $(i)$ is likely to be good if the ratio $A_{c} / A_{v} \gg 1$, e.g. in ${ }^{11} \mathrm{Be}(10: 1)$ or ${ }^{19} \mathrm{C}(18: 1)$ elastic scattering. It will also be true in Coulomb dominated processes when the core is charged and the valence particle neutral, as is usual for halo nuclei [16]. The validity of the adiabatic approximation for elastic scattering has been discussed extensively [17]. However, with our additional assumption $(i)$, it can be shown that the condition that the projectile excitation energy is small in comparison with the incident energy, when scattering through an angle $\theta$, is $4\left(A_{v} / A_{c}\right) \sin ^{2}(\theta / 2) \ll 1$. This suggests that the adiabatic assumption will be good for any incident energy and scattering angle provided $A_{v} / A_{c}$ is sufficiently small.

\subsection{Adiabatic three-body wavefunction}

Assuming $V_{v}=0$, the adiabatic approximation to the three-body wave function for the projectile, with ground state $\Phi_{0}(\boldsymbol{r})$ and binding energy $-\varepsilon_{0}$, incident with momentum $\boldsymbol{k}$ in the c.m. frame satisfies

$$
\left[T_{\boldsymbol{R}}+V_{c}\left(\boldsymbol{R}-\alpha_{v c} \boldsymbol{r}\right)-E_{0}\right] \Psi_{\boldsymbol{k}}^{(+) \mathrm{Ad}}(\boldsymbol{r}, \boldsymbol{R})=0
$$

The core-valence particle Hamiltonian has been replaced by $-\varepsilon_{0}$ under the assumption that the relative energies excited $\varepsilon \ll E$, the incident energy. Here $\boldsymbol{R}$ and $\boldsymbol{r}$ are the target-projectile and core-valence particle separations, with $T_{\boldsymbol{R}}$ the corresponding kinetic energy operator. $\alpha_{v c}=A_{v} /\left(A_{v}+A_{c}\right)$ so $\boldsymbol{R}_{c}=\boldsymbol{R}-\alpha_{v c} \boldsymbol{r}$ is the target-core separation. $E_{0}=E+\varepsilon_{0}=\hbar^{2} K^{2} / 2 \mu$ is now the incident c.m. kinetic energy and $\mu$ is the projectiletarget reduced mass.

Due to the adiabatic assumption, $\boldsymbol{r}$ is a parameter in Eq. (17) and the equation is simplified by transforming to variable $\boldsymbol{R}_{c}$. The key to the following analysis is to recognise [9] that the required solution of Eq. (17) is

$\Psi_{\boldsymbol{k}}^{(+) \operatorname{Ad}}(\boldsymbol{r}, \boldsymbol{R})=\Phi_{0}(\boldsymbol{r}) \exp \left(i \alpha_{v c} \boldsymbol{k} \cdot \boldsymbol{r}\right) \chi_{\boldsymbol{k}}^{(+)}\left(\boldsymbol{R}_{c}\right)$

where $\chi_{\boldsymbol{k}}^{(+)}$, the scattering wavefunction distorted by potential $V_{c}$, describes the scattering of the projectile, assumed pointlike and which cannot break-up, from this potential. We stress that the three-body wave function Eq. (18) retains break-up components

It follows immediately that, in the adiabatic approximation, the elastic scattering transition amplitude from state $\boldsymbol{k}$ to $\boldsymbol{k}^{\prime}$ now factorises as

$T_{e l}\left(\boldsymbol{k}^{\prime}, \boldsymbol{k}\right)=F(\boldsymbol{Q})\left\langle\boldsymbol{k}^{\prime}\left|V_{c}\right| \chi_{\boldsymbol{k}}^{(+)}\right\rangle=F(\boldsymbol{Q}) T_{p t}\left(\boldsymbol{k}^{\prime}, \boldsymbol{k}\right)$.

The second factor here is just the transition amplitude $T_{p t}$ for a point projectile scattering from $V_{c}$. The effects of projectile break-up and structure are therefore contained entirely within the first term, the form factor

$F(\boldsymbol{Q})=\int d \boldsymbol{r}\left|\Phi_{0}(\boldsymbol{r})\right|^{2} \exp (i \boldsymbol{Q} \cdot \boldsymbol{r})$

where (ignoring the weak binding effects) $\boldsymbol{Q}=\alpha_{v c}\left(\boldsymbol{k}-\boldsymbol{k}^{\prime}\right)$ is the momentum transfer to the valence particle in the scattering. 
This remarkable result means that, even in the presence of strong continuum channel coupling, the elastic scattering differential cross section of the composite takes the product form

$\left(\frac{d \sigma}{d \Omega}\right)_{e l}=|F(\boldsymbol{Q})|^{2} \times\left(\frac{d \sigma}{d \Omega}\right)_{p t}$,

where $(d \sigma / d \Omega)_{p t}$ is the point projectile cross section for scattering by the core-target interaction. Eq. (20) clarifies at which scattering angles and incident energies that a halo, of a given size and structure, can be observed as a deviation from the scattering expected due to point projectile scattering. Equation (21) looks like factorisations which arise when using Born approximation theories. We stress however that the present analysis does not involve Born approximation in any sense and that breakup effects are included to all orders, within the adiabatic approximation.

\subsection{Application to ${ }^{11}$ Be elastic scattering}

${ }^{11} \mathrm{Be}$ is a good example of a binary, ${ }^{10} \mathrm{Be}+\mathrm{n}$, single neutron halo nucleus, with a small $A_{v} / A_{c}$ ratio. It therefore provides a useful test case, and one for which there are some preliminary elastic scattering data available [18] with which to make a first assessment of the quantitative accuracy of the theory.

Calculation of the quantity $(d \sigma / d \Omega)_{p t}$ for the projectile is clearly highly constrained by, and closely related to, experimental data for the projectile core-target elastic scattering. This data, at the same energy per nucleon, is needed to constrain $V_{c}$. For the ${ }^{11} \mathrm{Be}+{ }^{12} \mathrm{C}$ system there are measured small angle elastic scattering data for both the ${ }^{10} \mathrm{Be}$ core and the ${ }^{11} \mathrm{Be}$ composite at similar energies per nucleon, 59.4 MeV/A and 49.3 MeV/A, respectively. Ideally these data are required at the same energy per nucleon. Fitting the ${ }^{10} \mathrm{Be}+{ }^{12} \mathrm{C}$ data allows us to obtain an estimate of $V_{c}$. In this case

$$
\begin{aligned}
V & =123.0 \mathrm{MeV}, \quad r_{V}=0.75 \mathrm{fm}, \quad a_{V}=0.80 \mathrm{fm}, \\
W & =65.00 \mathrm{MeV}, \quad r_{W}=0.78 \mathrm{fm}, \quad a_{W}=0.80 \mathrm{fm},
\end{aligned}
$$

where the potentials have volume real and imaginary Wood-Saxon terms and the radius parameters are scaled by $10^{1 / 3}+12^{1 / 3}$.

It is the formfactor $|F(Q)|^{2}$, which reflects the modifications to the scattering due to the composite (halo) nature of the projectile. For the ${ }^{11} \mathrm{Be}$ wavefunctions and formfactors we assume $2 s_{1 / 2}$ neutron single particle states, with separation energy $0.504 \mathrm{MeV}$. These are calculated in central Wood-Saxon potentials. We assume a fixed ${ }^{10} \mathrm{Be}$ core rms matter radius of $2.28 \mathrm{fm}$ and, by changing the binding potential geometry, obtain ${ }^{11} \mathrm{Be}$ with different rms matter radii and $|F(Q)|^{2}$; arising only from differences in the range of the neutron-core relative motion.

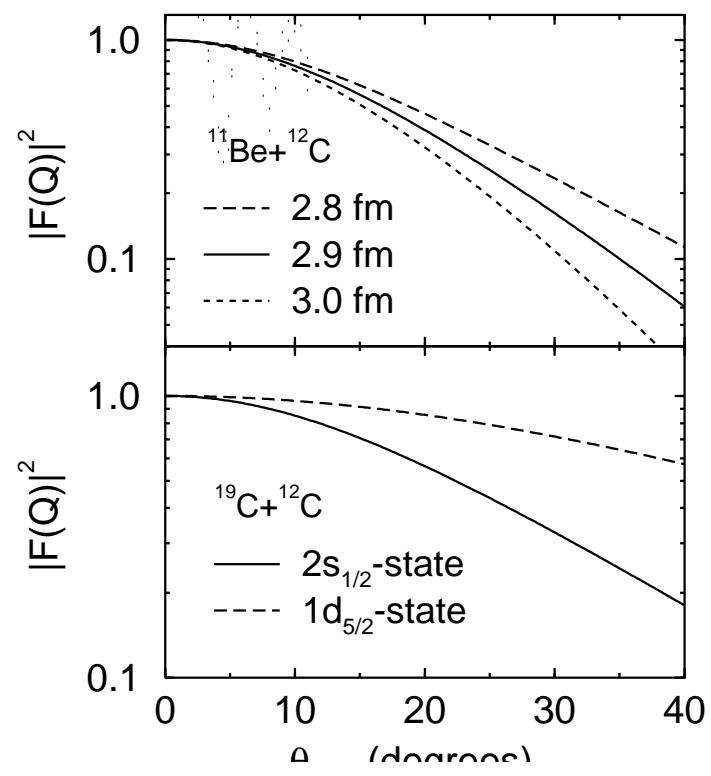

Figure 5. Calculated formfactors for ${ }^{11} \mathrm{Be}$ and ${ }^{19} \mathrm{C}$ scattering. 
These formfactors are shown in Figure 5 as a function of c.m. angle, for ${ }^{11} \mathrm{Be}+{ }^{12} \mathrm{C}$ scattering at $49.3 \mathrm{MeV} / \mathrm{A}$, for the ${ }^{11} \mathrm{Be}$ rms radii indicated. The formfactors demonstrate clearly the expected sensitivity of elastic scattering to the halo properties and the significant sensitivity to the ${ }^{11} \mathrm{Be}$ rms radius, i.e. the assumed rms separation of the valence and core particles.

Using the core interaction $V_{c}$ deduced above, the calculated $(d \sigma / d \Omega)_{p t}$ for point ${ }^{11} \mathrm{Be}$ scattering (long dashed curve) is compared, in Figure 6, with the experimental ${ }^{11} \mathrm{Be}+{ }^{12} \mathrm{C}$ scattering data at 49.3 $\mathrm{MeV} / \mathrm{A}[18]$. The calculated formfactor, for a ${ }^{11} \mathrm{Be}$ rms radius of $2.90 \mathrm{fm}$, is shown by the dashed-dotted curve. The product of these factors is the predicted adiabatic model elastic cross section, and is shown by the solid curve. The qualitative features of the data are well described showing the extent of the sensitivity of the elastic scattering data to the halo degrees of freedom.

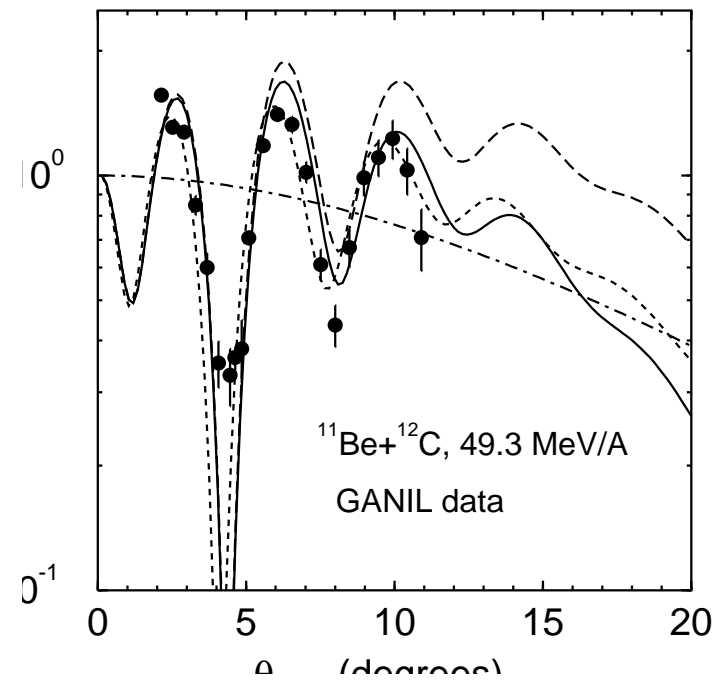

Figure 6. Calculated and measured elastic ${ }^{11} \mathrm{Be}+{ }^{12} \mathrm{C}$ scattering at $49.3 \mathrm{MeV} / \mathrm{A}$.

An obvious next question is: What is the importance (quantitatively) of the neglected valence neutron-target interaction and does its inclusion destroy the simple formfactor picture of the $V_{v}=0$ limit? Our treatment of a three-body system allows full quantum mechanical calculations, which use the adiabatic assumption but also include both $V_{v}$ and $V_{c}$ to be performed. For $V_{v}$ we take that neutron- ${ }^{12} \mathrm{C}$ interaction tabulated in [19].

Figure 6 also shows the full adiabatic calculation (short dashed curve) which includes $V_{v}$. The effects, while not entirely negligible, are seen to be rather small in comparison with the differences from the point case. To reinforce this point, in Figure 7 we show full adiabatic calculations (including $V_{v}$ ) for the three ${ }^{11} \mathrm{Be}$ wavefunctions considered here. Also shown are the corresponding formfactors of Figure 5. The upper curves show the results of dividing each full calculation by its respective formfactor. The essential coincidence of the three curves shows that the dependence of the cross section on the halo degrees of freedom through the formfactor persists even in the presence of the neutron interaction in this case.

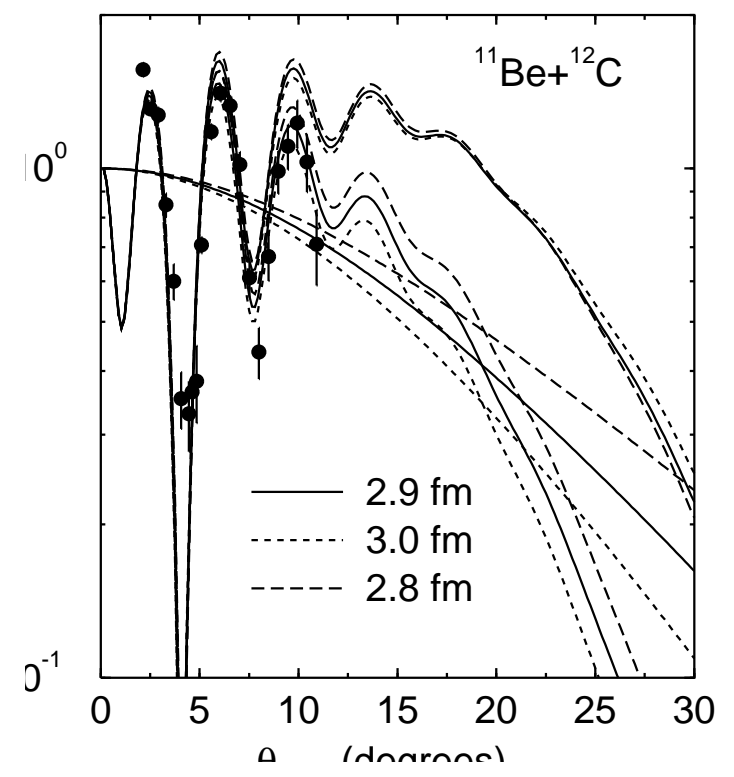

Figure 7. Full adiabatic calculations of elastic ${ }^{11} \mathrm{Be}+{ }^{12} \mathrm{C}$ scattering at 49.3 $\mathrm{MeV} / \mathrm{A}$. 


\subsection{Predictions for ${ }^{19} \mathrm{C}$ elastic scattering}

For ${ }^{19} \mathrm{C}$ the ground state structure is presently more uncertain, but the measured neutron separation energy of $0.240 \mathrm{MeV}$ and breakup momentum distributions [20] suggest a possible halo state. There are speculations of this being a pure $2 s_{1 / 2}$ state, a $1 d_{5 / 2}$ state, or a linear combination of such configurations [21]. Such structures would lead to different formfactors for elastic scattering.

There are no experimental elastic scattering data currently available, but the theory developed here allows simple predictions of the expected structure effects, through the structure formfactors presented above. Assuming the same potential parameterisation as for ${ }^{10} \mathrm{Be}$, above, and computing the $|F(Q)|^{2}$ which result from a pure $2 s_{1 / 2}$ (solid curve) and $1 d_{5 / 2}$ (dashed curve) neutron single particle state, we compare the predicted elastic scattering for ${ }^{19} \mathrm{C}+{ }^{12} \mathrm{C}$ at $30 \mathrm{MeV} / \mathrm{A}$, Figure 8 . There is about a factor of 2 difference expected in the calculated cross sections at $20^{\circ}$ due entirely to the formfactor, Figure 5. Data for the core and composite systems would be very interesting in assessing the presented theoretical model and the projectile structure further.

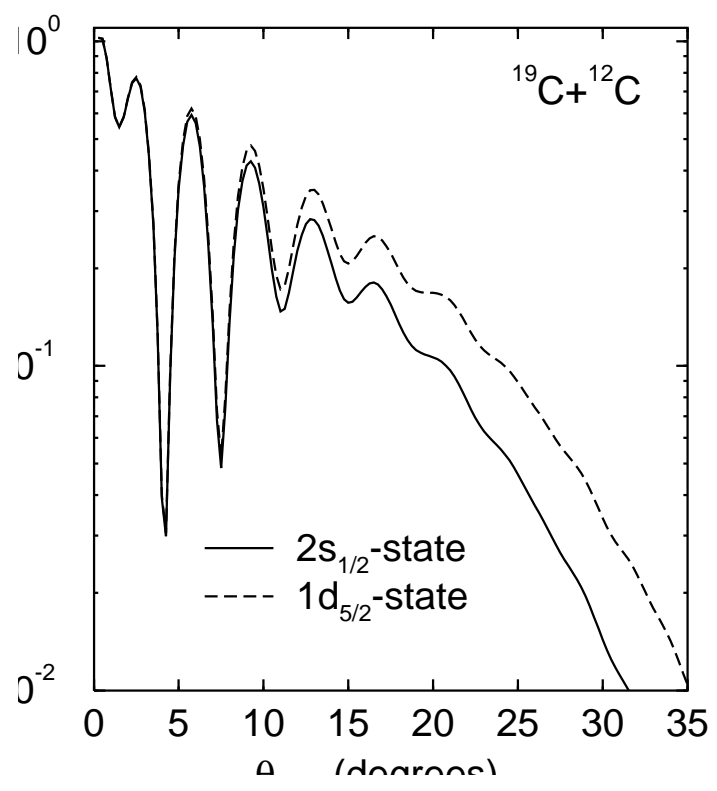

Figure 8. Elastic ${ }^{19} \mathrm{C}+{ }^{12} \mathrm{C}$ scattering at 30 $\mathrm{MeV} / \mathrm{A}$ assuming a $2 s_{1 / 2}$ or $1 d_{5 / 2}$ neutron single particle state.

\section{SUMMARY AND CONCLUSIONS}

In this paper we have considered the way in which the nucleus-nucleus total reaction cross section and elastic scattering differential cross section angular distributions reflect the size of the projectile nucleus. Specifically, we have considered the case where the projectile is a loosely bound composite with a well developed core and valence particle structure, such as a halo nucleus. We have considered the optical limit and few-body approaches to the calculation of reaction cross sections, making use of a simple binary cluster model to clarify the sensitivity of the cross sections calculated in these models to the sizes and nucleon clustering present within the system. Use of the OL theory is shown to consistently overestimate the calculated cross section and hence underestimate the nuclear size determined from experimental data. An outstanding disagreement between experiment and theory, in the case of the ${ }^{6} \mathrm{Li}$ and ${ }^{6} \mathrm{He}$, systems is discussed.

We have also shown that under certain conditions complementary information on the composite projectile size could be obtained from precision elastic scattering measurements at lower incident energies. We have shown that halo nucleus elastic scattering is strongly affected by breakup channels and the size of the halo. These effects reveal themselves simply, as a formfactor which multiplies the cross section due to point particle scattering 
by the interaction due to the projectile core, and which depends only on the valence particle ground state wavefunction. The core interaction can itself be highly constrained by high quality data for the core system. In final quantitative studies, it will probably be important to include also the interaction of the valence particle(s), but, the insight and simplicity revealed by the adiabatic model has been shown to persist in this case also. Additional experimental data are needed to assess these theoretical ideas further.

The authors gratefully acknowledge the contributions of Dr Ian Thompson, for providing and discussing the three-body wavefunctions for ${ }^{6} \mathrm{Li}$ and ${ }^{6} \mathrm{He}$ used here, and of Dr M.D. Cortina-Gil and Dr P. Roussel-Chomaz, GANIL, Caen, for the use of their experimental data for ${ }^{10,11}$ Be elastic scattering.

\section{REFERENCES}

1. I. Tanihata et al., Phys. Lett. B160 (1985) 380.

2. I. Tanihata et al., Phys. Lett. B206 (1988) 592.

3. R.J. Glauber, in Lectures in Theoretical Physics, ed. W.E. Brittin (Interscience, N.Y., 1959) Vol. 1, p. 315.

4. W. Czyz and L.C. Maximon, Ann. Phys. (N.Y.) 52 (1969) 59; P.J. Karol, Phys. Rev. C 11 (1974) 1203.

5. Victor Franco and Girish K. Varma, Phys. Rev. C 18 (1978) 349.

6. J.S. Al-Khalili and J.A. Tostevin, Phys. Rev. Lett. 76 (1996) 3903.

7. J.S. Al-Khalili, J.A. Tostevin and I.J. Thompson, Phys. Rev. C 54 (1996) 1843.

8. J.A. Tostevin and J.S. Al-Khalili, Nucl. Phys. A616 (1997) 418c.

9. R.C. Johnson, J.S. Al-Khalili and J.A. Tostevin, Elastic scattering of halo nuclei, Phys. Rev. Lett., in press.

10. S.K. Charagi and S.K. Gupta, Phys. Rev. C 41 (1990) 1610.

11. L. Ray, Phys. Rev. C 20, (1979) 1857.

12. I. Tanihata et al., Phys. Rev. Lett. 55 (1985) 2676.

13. I.J. Thompson, private communication.

14. H. deVries et al., At. Data and Nucl. Data Tables, 36 (1987) 495.

15. H. Amakawa, S. Yamaji, A. Mori and K. Yazaki, Phys. Lett. B82 (1979) 13; I.J. Thompson, Computer program ADIA, Daresbury Laboratory Report, 1984, unpublished.

16. J.A. Tostevin et al., Coulomb breakup of light composite nuclei, submitted to Phys. Lett.

17. N. Austern et al., Physics Reports 154 (1987) 125.

18. P. Roussel-Chomaz, private communication, 1996.

19. J.S. Al-Khalili, I.J. Thompson and J.A. Tostevin, Nucl. Phys. A581 (1995) 331.

20. D. Bazin et al., Phys. Rev. Letts. 74 (1995) 3569; F.M. Marqués et al., Phys. Lett. B381 (1996) 407.

21. D. Ridikas, M.H. Smedberg, J.S. Vaagen and M.V. Zhukov, Europhys. Lett. 37 (1997) 385. 